\title{
Evaluation of the additive effect of bevacizumab eye drops to mitomycin $C$ in primary pterygium
}

\begin{abstract}
Purpose: To assess the additive effect of postoperative Bevacizumab eye drops to MMC augmented bare sclera in cases of primary pterygium concerning the safety and recurrence rate.

Patients and methods: This prospective randomized controlled study included 20patients with bilateral primary pterygium. The right eye of each patient was treated by simple pterygium excision with bare sclera and MMC application followed by artificial tear eye drops for 4 weeks postoperative as placebo (group I included 20eyes) and served as the control group. The left eye of each patient was treated by simple excision with bare sclera and MMC followed by topical Bevacizumab eye drops for 4weeks postoperative (group II included 20eyes). Both eyes were operated upon in the same session. All subjects were examined on days $1,7,14$ and 30 and monthly for year to record any vascularization measured in $\mathrm{mm}$ or recurrence of pterygium measured horizontally from the limbus to its apex in the cornea.

Results: After 12 months of follow up, 3eyes (15\%) with recurrence were seen in group I which was not significantly different from group II with 2eyes (10\%). No corneal neovascularization in group II except in 7eyes of which only $1-2 \mathrm{~mm}$ neovessels growth in 5 eyes and one eye more than $3 \mathrm{~mm}$ and another eye $2-3 \mathrm{~mm}$ however in group I no vascularization in only 5 eyes with more than $3 \mathrm{~mm}$ neovessels in 3 eyes, 5 eyes with neovessels $2-3 \mathrm{~mm}$ and 7 eyes with $1-2 \mathrm{~mm}$ neovessels. Total P-Value concerning the presence and absence of vascularization between the two groups was 0.000805 which was highly significant.

Conclusion: Our study concluded that recurrence was not affected by the topical Bevacizumab with no statistically significant difference between the two groups; however, a statistically significant difference existed in the level of corneal neovascularization between the two groups suggesting that regression of neovessels is not a guarantee against recurrence of pterygium.
\end{abstract}

Keywords: primary pterygium, bevacizumab, mitomycin C
Volume 6 Issue 2 - 2017

\section{Sameh M Fouda}

Department of Ophthalmology, Zagazig University, Egypt

Correspondence: Sameh M Fouda, Ophthalmology Department, Zagazig University, Egypt, Tel 0020 I28492 I290, Email samehfouda@hotmail.com

Received: December 31, 2016 | Published: January 31, 2017
Abbreviations: VEGF, vascular endothelial growth factor; 5FU, 5 fluorouracil; MMC, mitomycin C; HTF's, human tenon fibroblasts

\section{Background}

Simple excision of pterygium, although very simple, is associated with variable postoperative recurrence rate which is especially high with bare sclera technique. ${ }^{1,2}$ Recurrence of pterygium could be attributed to episcleral fibroblasts proliferation and neovascularization secondary to the traumatic and inflammatory processes following pterygium surgery. Histologically, pterygium comprises a highly developed vascular neoformation network that lies in a body of hypertrophic and elastotic connective tissue and covered with an atrophic conjunctival epithelium. Several active angiogenic and epithelial growth factors have been shown to be drastically increased in pterygium tissue; the most crucial molecule among these factors is the vascular endothelial growth factor (VEGF). The abundant expression of the vascular endothelial growth factor (VEGF) in pterygia suggests that anti-VEGF therapy may induce regression of blood vessels in pterygia or prevent its recurrence after excision. ${ }^{3,4}$ Mutation in P53 gene on chromosome 17 with induced changes in the expression of Vascular Endothelium Growth Factor (VEGF) was suggested as a presumed etiology for pterygium recurrence. ${ }^{5}$

Several adjunctive agents have been tried to reduce the rate of recurrence, among them 5 Fluorouracil (5FU) and Mitomycin $\mathrm{C}$ (MMC) are still in use. MMC is an alkylating agent that acts by cross-linking the DNA of fibroblast and hence preventing it from proliferation and differentiation into myofibroblast in the episclera. ${ }^{6,7}$ Bevacizumab (Avastin; Genetech, San Fransisco, CA, USA) is a recombinant humanized monocolonal immunoglobulin G1 antibody that acts directly to inhibit VEGF. It was known to inhibit corneal neovascularization both in rat models and in humans. ${ }^{89}$ This study was conducted to assess the additive effect of postoperative Bevacizumab eye drops to MMC augmented bare sclera in cases of primary pterygium concerning the safety and recurrence rate.

\section{Patients and methods}

This prospective randomized controlled study included 20patients with bilateral primary pterygium. Written consent was obtained from the patients and included an explanation of the study and agreement to have a photo of the eye area only taken. This study work follows the recommendations of the Ethics Committee of Zagazig university 
(ZU-IRB) which conforms to the provisions of the Declaration of Helsinki in 1995 (as revised in Edinburgh 2000). The sample size was calculated to be 40eyes, the calculation was done using Epi-Info (Epidemiological Information Package) software version 6.1. The patients were selected from the patients attending the outpatient clinic of ophthalmology department of Zagazig University Hospital during the period from November 2013 to December 2014.

To ensure matching between the study groups, patients with bilateral pterygia only were included in the study and the right eye was randomly selected to be treated with MMC only while the left eye was randomly selected to be treated with both MMC and Bevacizumab. The right eye of each patient was treated by simple pterygium excision with bare sclera and MMC application followed by artificial tear eye drops for 4 weeks postoperative as placebo (group I included 20eyes) and served as the control group. The left eye of each patient was treated by simple excision with bare sclera and MMC followed by topical Bevacizumab eye drops for 4 weeks postoperative (group II included 20eyes). Both eyes were operated upon in the same session.

Preoperative assessment of the patients included complete history taking with special emphasis on previous ocular surgeries or systemic diseases especially connective tissue disorders. Complete ophthalmological examination including: visual Acuity, local slit lamp biomicroscopy with special stress on pterygium size and depth into the cornea and intraocular pressure measurement. Only patients with bilateral primary pterygium were enrolled in this study. Patients with previous pterygium or any other ocular surgeries, patients on ocular medications and patients with known connective tissue disorders were excluded from the study.

\section{Surgical technique}

Benoxinate $\mathrm{Hcl} 0.4 \%$ was instilled before surgery, lid speculum was inserted and $0.5 \mathrm{ml}$ mixture of local anesthetic lidocaine HCL 20mg/ $\mathrm{ml}$ and epinephrine $0.0125 \mathrm{mg} / \mathrm{ml}$ was injected subconjunctivally in the pterygium area using a 25-gauge needle. keratectomy was done with beaver No 15 blade and was started $1 \mathrm{~mm}$ in front of the apex of pterygium till the limbus where dissection and excision of the body was done. Tenon's capsule was excised with cauterization of the episcleral vessels. A cellulose sponge $3 \mathrm{~mm} \times 4 \mathrm{~mm}$ containing $0.2 \mathrm{mg} /$ $\mathrm{ml}$ MMC was applied directly to the scleral bed for 2 minutes then irrigated with $30 \mathrm{ml}$ of normal saline solution to clear MMC residues. Closure of the conjunctiva by $8 / 0$ Vicryl sutures was done leaving $2-3 \mathrm{~mm}$ bare scleral area nasal to the limbus. The same technique was done in the second eye and patching of both eyes after the application of topical tobramycine and dexamethazone eye ointment (Tobradex ${ }^{\circledR}$, Alcon Inc. USA ).

\section{Postoperative care}

The right eye of each patient received topical tobramycine and dexamethazone (Tobradex ${ }^{\circledR}$, Alcon Inc. USA) eye drops 4 times a day, topical tobramycine and dexamethazone (Tobradex $\AA$, Alcon Inc. USA) ointment at bed time and artificial tears eye drops 4times a day for 4 weeks duration, the left eye received the same treatment as the right eye but instead of artificial tears Bevacizumab eye drops $10 \mathrm{mg} / \mathrm{ml}(1 \%)$ was used 4times a day (in an identical bottle to the artificial tears eye drops bottle) for the same duration. The dose was calculated after Motarjemizadeh et al. ${ }^{10}$ who suggested that $10 \mathrm{mg} /$ $\mathrm{ml}$ concentration of topical bevacizumab was a more effective dose for adjuvant pterygium therapy than $5 \mathrm{mg} / \mathrm{ml}$ dose. Bevacizumab was prepared and diluted with $0.9 \%$ normal saline under aseptic conditions and stored at $2-8^{\circ} \mathrm{C}$ as recommended by the manufacturer, the patients were instructed to dispose and change the eye drops bottle after one month and to place it in the refrigerator all the time.

All subjects were examined on days 1, 7, 14 and 30 and monthly for one year, in each visit slit lamp biomicroscopy was performed to record any neovascularization measured in $\mathrm{mm}$ or recurrence of pterygium measured horizontally from the limbus to its apex in the cornea, also any patient complaints were recorded especially those suggestive of any toxic systemic or ocular side effects as pain and burning sensation. The study was performed in a single-blinded way in which the patient did not know the type of eye drops used in each eye. The recurrence was defined as any new fibrovascular tissue growth observed on slit lamp examination beyond the limbus. Statistical analysis of the data was performed using the $\mathrm{Z}$ and Chi square tests.

\section{Results}

This study included 16 males $(80 \%)$ and 4 females (20\%). Age of the patients ranged from 39 to 68years with a mean age of 53.6years. According to the time spent outdoors in the patients, 6patients $(30 \%)$ were exposed to the sun for about 4-8hours daily while the remaining 14 patients $(70 \%)$ were exposed to more than 8 hours per day. Sixteen patients $(80 \%)$ had their pterygium duration of more than 2 years and 4 patients $(20 \%)$ had duration less than 2 years. All eyes had pterygia more than $2 \mathrm{~mm}$ invasion inside the cornea. The postoperative results after 12 months of follow up revealed that: In the first group, treated postoperatively by placebo eye drops, only 3eyes $(15 \%)$ showed recurrence and that was not significantly different from the bevacizumab group which showed 2 eyes with recurrence $(10 \%)$. Table 1 shows the post-operative recurrence in the two groups.

Regarding corneal neovascularization, eyes were classified into 4categories according to the extent of neovascular invasion beyond the limbus. The results are represented in Figure 1. Apart from the $1-2 \mathrm{~mm}$ corneal neovascularization category in which the control group had 7 eyes (35\%) versus 5eyes (25\%) in the bevacizumab group, there were significant difference between the two studied groups in all other neovascularization categories. The total P-value concerning the presence and absence of vascularization was 0.000805 which is highly significant. There were no eyes with toxic side effects as pain or burning sensation in both groups. Figure 1 shows the postoperative neovascularization in the 2groups.

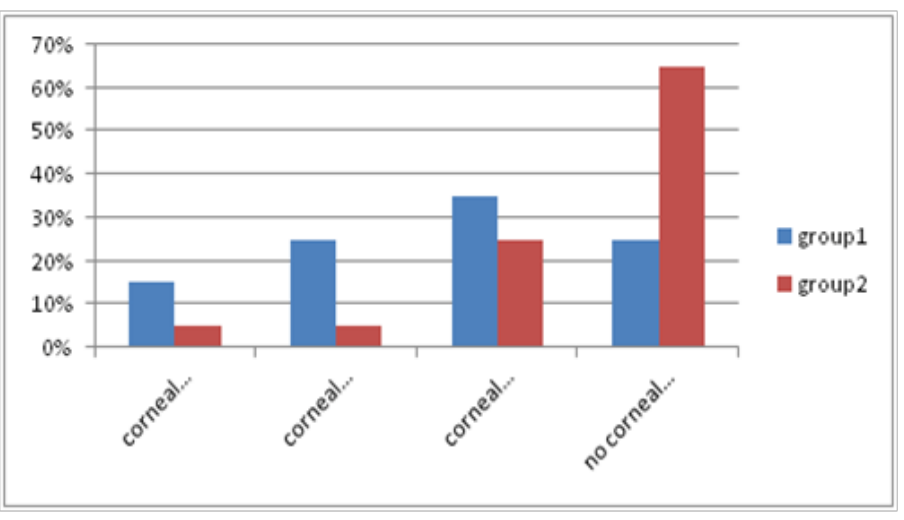

Figure I The incidence of neovascularization among the two groups. 
Table I The postoperative recurrence rate in the two groups

\begin{tabular}{llll}
\hline & Group I & Group 2 & P value \\
\hline Total & 20 & 20 & 0.6353 \\
Recurrence: Number & 3 & 2 & \\
Percentage & $15 \%$ & $10 \%$ & \\
\hline
\end{tabular}

\section{Discussion}

Recurrence of pterygium following primary pterygium excision is a frustrating complication to both the patient and the surgeon. And it is associated with complications such as cosmetic problems, inflammatory changes, and oculomotor disorders or diplopia resulting from adhesion. Many wound modulating agents have been used in a trial to minimize recurrence, including irradiation, antimetabolites namely; 5-fluorouracil and mitomycin C. They all carried some complications such as scleral thinning, corneal damage and consequences of radiation exposure. It has been well-established that pterygium is composed of fibrovascular tissue and the pterygium formation and progression require neovascularization, suggesting that the vascular endothelial growth factor (VEGF) is involved in the pathogenesis of pterygia. ${ }^{11}$

With the emergence and availability of antivascular endothelial growth factors (anti-VEGF), they were suggested as a possible adjunctive therapy for pterygium excision by decreasing the vascularity of newly formed blood vessels, hence decreasing the recurrence rate. ${ }^{12,13}$ Intralesional injection of Bevacizumab was found by Fallah et al. ${ }^{14}$ to decrease pterygium size. Two separate studies conducted by El Shafie et al. ${ }^{15}$ and Singh et al. ${ }^{16}$ have concluded that preoperative administration of subconjunctival injection of Bevacizumab before the pterygium excision with conjunctival autograft is useful in the treatment of patients with primary pterygium without local or systemic adverse effects. Another study by Alhammami et al. ${ }^{17}$ concluded that subconjunctival Bevacizumab is of benefit in cases of recurrent pterygium. The subconjunctival injection carries the potential risk of systemic complications such as gastrointestinal perforation, hypertension, arteriolar hemorrhage, impaired wound healing, bleeding, endophthalmitis, and arterial thromboembolic events Therefore, it has been suggested that topical instillation is the best route of Bevacizumab administration because it provides both safety and efficacy. ${ }^{18,19}$

The dose of topical bevacizumab was debated by many authors. An in vitro study conducted by Park et al. ${ }^{20}$ found that both metabolic activity and viability of primary and recurrent pterygium human Tenon fibroblasts (HTF's) are inhibited by bevacizumab in a dosedependent manner, especially at concentrations greater than $7.5 \mathrm{mg} /$ $\mathrm{ml}$. However, primary pterygium HTFs shows reduction in cellular density at a bevacizumab concentration of $5.0 \mathrm{mg} / \mathrm{ml}$. Motarjemizadeh et al. ${ }^{10}$ evaluated the impact of topical administration of Bevacizumab on pterygium recurrence, they found that subjects who had received placebo were about 5 times more likely to suffer from pterygium recurrence than patients who had been treated by $5 \mathrm{mg} / \mathrm{ml}$ concentration of topical Bevacizumab. Moreover, no recurrences at all were observed in the patients receiving a concentration of $10 \mathrm{mg} /$ $\mathrm{ml}$ suggesting that it is the optimum concentration needed to prevent recurrence.
On the other hand, Hwang and Choi ${ }^{21}$ conducted a comparative study between topical $0.02 \%$ mitomycin $\mathrm{C}$ and $0.05 \%$ cyclosporine and topical $2.5 \%$ bevacizumab after surgery. They stated that lower recurrence rates were encountered in the mitomycin $\mathrm{C}$ and cyclosporine groups when compared to their control group, whereas the bevacizumab group showed no difference in the recurrence rate. Moreover, Razeghinejad and Banifatemi. ${ }^{22}$ found that Bevacizumab had no significant effect on the recurrence rate of pterygium Although reduction in the frequency of fibrovascular tissue crossing the limbus in the bevacizumab group to half that of the BSS group, the difference was not statistically significant. To our best knowledge, this is the first eye- to -eye study that evaluates the additive effect of Bevacizumab eye drops to the MMC in cases of primary pterygium regarding both recurrence and corneal neovascularization. The lack of a detectable intergroup difference may be due to small sample size. Furthermore, the delivered dose might have been too small to reach the desired concentration to inhibit a continuously generated pool of VEGF. Moreover, the presence of abundant conjunctival vessels increases the rate of systemic drug absorption and thus reducing the amount of locally available drug.

\section{Conclusion}

Our results showed that recurrence was not affected by the topical Bevacizumab with no statistically significant difference between our two groups, however, a statistically significant difference existed in the level of corneal neovascularization between the two groups suggesting that regression of neovessels is not a guarantee against recurrence of pterygium. Further studies are needed to evaluate the effectiveness of topical bevacizumab and other anti- VEGF in the prevention of recurrence of primary pterygia.

\section{Acknowledgments}

None.

\section{Conflicts of interest}

The authors declare that there is no conflict of interest.

\section{Funding}

None.

\section{References}

1. Hoffman RS, Power WJ. Current options in pterygium management. Int Ophthalmol Clin. 1999;39(1):15-26.

2. Hill JC, Maske R. Pathogenesis of pterygium. Eye. 1989;3(2):218-226.

3. Gordon CR, Rojavin Y, Patel M, et al. A review on bevacizumab and surgical wound healing: An important warning to all surgeons. Ann Plast Surg. 2009;62(6):707-709.

4. Mauro J, Foster CS. Pterygia: pathogenesis and the role of subconjunctival bevacizumab in treatment. Semin Ophthalmol. 2009;24(3):130-134.

5. Bazzazi N, Ramezani A, Rabiee MA. A comparative study of conjunctival autograft and minimally invasive pterygium surgery in primary pterygia. PakJ Biolog Sci. 2010;13(8):409-412.

6. Singh G, Wilson MR, Foster CS. Mitomycin eye drops as treatment for pterygium. Ophthalmology. 1988;95(6):813-820. 
7. Frucht-Pery J, Siganos CS, Ilsar M. Intraoperative application of topical mitomycin-C for pterygium surgery. Ophthalmology. 1996;103(4):674-677.

8. Hosseini H, Nejabat M, Khalili MR. Bevacizumab (Avastin) as a potential novel adjunct in the management of pterygia. Med Hypotheses. 2007;69(4):925-927.

9. Ferrara N, Hillan KJ, Novotny W. Bevacizumab (Avastin), a humanized anti-VEGF monoclonal antibody for cancer therapy. Biochem Biophys Res Commun. 2005;333(2):328-335.

10. Motarjemizadeh Q, Aidenloo NS, Sepehri S. A comparative study of different concentrations of topical bevacizumab on the recurrence rate of excised primary pterygium: a short-term follow-up study. Int Ophthalmol. 2006;36(1):63-71.

11. Sudhalkar A, Sudhalkar A. Outcomes of Post-Operative Topical Bevacizumab in Primary Pterygium Surgery: A Case Series. J Clin Exp Ophthalmol. 2012;3:243.

12. Fallah MR, Khosravi K, Hashemian MN, et al. Efficacy of topical bevacizumab for inhibiting growth of impending recurrent pterygium. Curr Eye Res. 2010;35(1):17-22.

13. Wu PC, Kuo HK, Tai MH, et al. Topical bevacizumab eyedrops for limbal-conjunctival neovascularization in impending recurrent pterygium. Cornea. 2009;28:103-104.

14. Fallah Tafti MR, Khosravifard K, Mohammadpour M, et al. Efficacy of intralesional bevacizumab injection in decreasing pterygium size. Cornea. 2011;30(2):127-129.
15. El Shafie AM, Mohamed AS, Sayed MF. The outcome of preoperative subconjunctival bevacizumab injection in pterygium surgery. $J$ Egypt Ophthalmol Soc. 2014;107:113-116.

16. Singh P, Sarkar L, Sethi HS, et al. A randomized controlled prospective study to assess the role of subconjunctival bevacizumab in primary pterygium surgery in Indian patients. Indian J Ophthalmol. 2015;63(10):779-784.

17. Alhammami H, Farhood Q, Shuber H. Subconjuctival bevacizumab injection in treatment of recurrent pterigym. $J$ Clin Exp Ophthalmol. $2013 ; 4: 267$

18. Stevenson W, Cheng SF, Dastjerdi MH, et al. Corneal neovascularization and the utility of topicalVEGF inhibition: ranibizumab (Lucentis) vs bevacizumab(Avastin). Ocul Surf. 2012;10(2):67-83.

19. Chen JJ, Ebmeier SE, Sutherland WM, et al. Potential penetration of topical ranibizumab (Lucentis) in the rabbit eye. Eye (Lond). 2011;25(11):504-511.

20. Park YM, Kim CD, Lee JS. Effect of Bevacizumab on Human Tenon's Fibroblasts Cultured from Primary and Recurrent Pterygium. Korean J Physiol Pharmacol. 2015;19(4):357-363.

21. Hwang S, Choi S. A comparative study of topical mitomycin c, cyclosporine, and bevacizumab after primary pterygium Surgery. Korean J Ophthalmol. 2015;29(6):375-381.

22. Razeghinejad MR, Banifatemi M. Subconjunctival bevacizumab for primary pterygium excision; a randomized clinical trial. J Ophthalmic Vis Res. 2014;9(1):22-30. 